\title{
Neuroprotective Efficacy of Maytenus emarginata (Wild.) Ding Hou Against Paraquat Induced Oxidative Stress and Neurotoxicity in Drosophila Model System
}

\author{
Leelaja Bhadravathi Chandrappa ${ }^{1}$, Panduranga Murthy Govinda Shetty ${ }^{2}$, , \\ Ravishankar Doddarasinakere Kempaiah ${ }^{3}$, Jayaramu Neelakantanalli ${ }^{4}$, \\ Dharshan Raj Chenna Govindaraju ${ }^{5}$, Rayankula Naidu ${ }^{6}$ \\ ${ }^{1}$ Department of Studies in Biochemistry and Molecular Biology, University of Mysore, Mysore, India \\ ${ }^{2}$ Maharaja Institute of Technology Thandavapura, Visvesvaraya Technological University, Mysore, India \\ ${ }^{3}$ Mahadeshwara Government First Grade College, University of Mysore, Kollegal, India \\ ${ }^{4}$ Department of Chemistry, Bharathi College, University of Mysore, Mysore, India \\ ${ }^{5}$ Pallikere Abdulla College of Engineering, Mangalore, India \\ ${ }^{6}$ Herbal Drug Research Laboratory, Hamsageetha Research Foundation, Tumkur, India
}

Email address:

pranga.leeja@gmail.com (P. M. G. Shetty)

*Corresponding author

To cite this article:

Leelaja Bhadravathi Chandrappa, Panduranga Murthy GovindaSetty, Ravishankar Doddarasinakere Kempaiah, Jayaramu Neelakantanalli, Dharshan Raj Chenna Govindaraju, Rayankula Naidu. Neuroprotective Efficacy of Maytenus emarginata (Wild.) Ding Hou Against Paraquat Induced Oxidative stress and Neurotoxicity in Drosophila Model System. American Journal of Applied Chemistry.

Vol. 7, No. 1, 2019, pp. 25-34. doi: 10.11648/j.ajac.20190701.13

Received: September 19, 2018; Accepted: March 26, 2019; Published: April 18, 2019

\begin{abstract}
An investigation has been made to find out the disease altering therapies against a somber ailment called 'early aging' which is due to the formation of free radicals during the natural course of metabolism, also act randomly and indiscriminately to damage the cell components. On the contrary, the natural antioxidants in dietetic practice can diminish these free radicals which can perturb further, the progression of these age-related neurodegenerative disorders such as Alzheimer's disease (AD), Parkinson's disease (PD), and Huntington's disease (HD) followed by Canine Cognitive dysfunction. As a consequence, a tribal medicinal plant; Maytenus emarginata (Wild.) Ding Hou (family- 'Celastraceae') was identified which is commonly called 'Thorny staff tree' a potent ethno-medicinal plant drug was opted during the interactions held with tribal healers. This candidate plant drug is being practiced as memory-enhancing factor in their tribal medicinal system at Biligirirangana Hills (B. R. Hills) of Chamarajanagara district, India. Hence, the biological screening with Methanol extract of Maytenus emarginata (MEM) was attempted initially for Physico-chemical, Phyto-chemical properties followed by in vitro, in vivo antioxidant and neuroprotective activities using Drosophila melanogaster (Oeragon K) strain adult male flies. In the result, the extract of M exhibited concentration dependent DPPH scavenging activity. The oxidative stress markers employed to access in vivo antioxidant property of $\mathrm{M}$ included lipid peroxidation products malondialdehyde (MDA) and hydroperoxide (HP), reduced glutathione (GSH). The modulatory effect of M on superoxide dismutase (SOD) and catalase (CAT) was also determined. The oxidative stress was induced by using paraquat at $15 \mathrm{mM}$. The concentration of extract for studies was fixed based on $\mathrm{LC}_{50}$ values. There was a significant demolition in the levels of MDA and HP in co-exposure of M with Par treated flies homogenate. The level of SOD and CAT was brought to near basal level in the homogenate of flies co-exposed with M and Par. In negative geotaxis assay it was found that $\mathrm{M}$ was able to rescue the flies significantly from deteriorating locomotors dysfunctions. The $\mathrm{M}$ extract exhibited significant modulatory activity in regulating oxidative stress markers and antioxidants in the fly homogenate. The extract $\mathrm{M}$ also exhibited significant antibacterial property against tested microbial strains. Therefore, the findings point out that, MEM plant drug was found to be most promising in its efficacy which may act as compelling antioxidant agent that can intensify the locomotors functions. Thus, the MEM plant drug therapy can regulate the occurrence of $\mathrm{CCD}, \mathrm{AD}, \mathrm{PD}$ and $\mathrm{HD}$ and the normal condition may be reinstalled in the biological system.
\end{abstract}


Keywords: Maytenus emarginata (Willd.) Ding Hou, Ethno-Medicinal Plant Drug, Tribal Healers, Pharmacognostic Property, Antioxidant Activity, Neuroprotective Activity, Drosophila Model System

\section{Introduction}

The life span of human being has come down to sixty years due to environmental dreadful conditions which are in turn caused by irrational anthropogenic activities. As a result, the early aging has been a serious disorder amongst human populace thus, it has become an innermost focus due to various quandaries occurred in the biological system. Owing to this, the free radicals are formed during the natural course of metabolism, also act intermittently and erratically to damage the cell components. Eventually, this multifold cellular damage due to accumulation of free radicals may contribute to the aging process. In the later part, the incidence of age-related neurodegenerative disorders such as Alzheimer's disease (AD), Parkinson's disease (PD), and Huntington's disease (HD) are elicited by the destructive role of free radicals in the human system. However, this turmoil condition has been characterized by adverse changes in learning and memory patterns in the age related neurodegenerative conditions which is mainly due to Canine Cognitive dysfunction (CCD). Consequently, the break through therapeutic approach against these neurodegenerative disorders has become a crucial and confronting issue in the present scenario. In the pace of rationalization, the previous reports explicitly suggest that, the oxidative damage is rapidly increases with age and unrestrained in both human and canine brain. Further, the preceding reports also demonstrated that, by augmenting the cell's antioxidant defenses with certain supernatural enzymes which can able to lessen the cellular damage caused by the action of free radicals. Correspondingly, the natural antioxidants in dietetic practice hold a great promise in tormenting the progression of these neurodegenerative disorders. Hence, the real need of the hour is to find out the plant based disease altering therapies against age-related neurodegenerative disorders [1].

Currently, the only system called 'Ayurveda' can able to meet the demands of herbal medicines against various ailments and diseases in the health care system. It is one of the efficient ancient systems of Indian medicine, which has defined a number of plants with therapeutic benefits for the treatment of neurodegenerative ailments, having antioxidant activities. But, on the serious consequences, the plant defined medicines with appropriate formula is lacking in the present practice of herbal medicines. On the contrary, the revitalization and rejuvenation healing therapy through ethno-medicinal practice by tribal healers or medicine men in their unique medicine system called 'Tribal medicine System' with superlative medicine formula against various serious illness and ailments are of great importance. The distinct ethno-medicine plant drugs with vital formula act inside the human body by modulating the nuero-endocrino-immune systems and have been found to be a rich source of antioxidants. The investigations on these undisclosed ethnomedicinal herbal formulas characterize a great deal of untapped reservoir of drugs and the structural diversity of their component molecules makes a valuable source of novel lead compounds for neuro-degeneration ailments [2, 3].

\subsection{Backdrop on Neuroprotection}

Neuroprotection is a broad term to cover any therapeutic strategy to prevent nerve cells called neurons from dying and it usually involves an intervention, either a drug or treatment. It is a mechanism used to protect neuronal injury or degeneration of CNS following acute disorders. The goal of neuro-protection is to limit neuronal dysfunction after injury and attempt to maintain the possible integrity of cellular interactions in the brain resulting in serene neural function. However, latterly, the study of antioxidants has drawn colossal attention due to diverse health implications. The central nervous system (CNS) will be defenseless in response to oxidative stress as a cause of high metabolic rate and deficient antioxidant system. The escalating totting up of experimental evidence confirms the partaking of reactive oxygen species in neurodegenerative diseases. Nevertheless, the antioxidants deactivate the free radicals by counteracting which leads to neutralization and are more effectual in suppressing or averting these disorders. Subsequently, Ayurveda, an ancient system of Indian medicine, has demonstrated a number of plant oriented drugs with therapeutic benefits for the treatment of neurodegenerative disease, encompassing antioxidant activities [4].

\subsection{Oxidative Stress and Antioxidant Agents}

An antioxidant is any substance that when present at low concentrations compared to oxidizable substance, significantly delays or prevents oxidation of that substrate. The oxidative stress can lead to cell and tissue injury. However, the free radicals that are generated during oxidative trauma those are formed during normal metabolism and thus involve in differing of human health and cause disease. Oxygen radicals may be formed as a by-product of enzymatic reactions in vivo. Oxidative stress is a cytotoxic consequence of such by products observed during neurodegeneration and ageing process [5].

Consequently, substantial numbers of drugs are developed from ethno-medicinal plants which are active against a number of ailments/diseases. The isolation of bio-active constituents found in a particular ethno-medicinal formula comprising individual and combination of potent herbs with a new fangled subsequent modification is a real need towards development of an efficacious drug [6]. Subsequently, in the 
literature study, it was also noticed that, there is a dearth of studies on its neuroprotective evaluation from this plant drug. Therefore, an attempt has been made here to appraise the herbal drug which is an infrequent ethno-Medicinal plant formula which is untouched in the literature. Hence, the screening of plant drug encompassing on Antioxidant and Antimicrobial properties which are in linking with Neuroprotective activity in Maytenus emarginata (Wild.) Ding Hou using Drosophila model system was carried-out.

\subsection{Ethnomedicines as Neuroprotective Agent}

Ethno-medicine means an herbal medicine formula prepared on the basis of traditional knowledge and the same being practiced by a specific ethnic group or tribal community living in particular bio-geographical locations which is unmarked with civilized society. This is said to be ethno-medicine practiced by ethnic group which is also called 'Community medicine'. Amongst Ayurveda, Sidda, Unani, the ethno-medicine remains a vital alternative source of medicine in rural parts of different countries and it is less known to the world today with some estimate indicating about more than $60 \%$ of the primary and serious ailments are being effectively treated in the tribal health care system which are evidenced in some developing countries such as, Nepal, Nigeria, Ghana, SriLanka, China and India [2, 3, 68].

Several natural products namely, active principle, lead molecules, chief chemical constituents derived from crude and fractionated extracts of identified ethno-medicinal plants, have been reported to be used in traditional medicine for various serious ailments such as, cancer, gangrene, wound healing, snake bite, skin $\&$ respiratory disorders followed by efficient utilization of herbal drug formula as neuroprotective, memory enhancing, and anti-ageing purposes respectively [1, 9, 10].

Consequently, the explicit studies have proved that certain non-nutritive chemicals in plants called secondary metabolites such as alkaloids, terpenoids and flavonoids possess incredible antioxidant properties. The lack of effective and widely applicable pharmacological treatments in the modern therapy for neurodegenerative disorders may explain a growing interest in the traditional medicines [11]. Therefore, some available scientific literature revealing neuroprotective action of ethno-medicinal plants are proposed to undertake the objectives with real need of the hour. However, a medicinal plant; Maytenus emarginata (Wild.) Ding Hou (family-'Celastraceae') has been identified based on the suggestions made by the tribal healers during interactions which are being practiced for memory-enhancing in their tribal medicinal system at Biligirirangana Hills (B. R. Hills) of Chamarajanagar district, Karnataka, India. During interaction with 'Tribal medicine men', it was noticed that, the plant crude extracts of the M. emarginata have been also used in the treatment for Plethora of medical ailments for nervous disorder, fever, inflammation, allergy, microbial infection, stomach disorder, rheumatoid arthritis and cancer etc.

\subsection{Maytenus emarginata (Wild.) -An Ethno-Medicinal Plant Drug}

Maytenus emarginata is an evergreen small tree that tolerates various types of stresses of the desert, locally known as "Kankero" in Hindi, "Thorny staff tree" in English and it is found in all major groups of biogeographical parts of India [12]. The crude plant extracts of the plant drug formula was being prepared using goat milk and raw honey by tribal medicine men in their customary medicine system.

The different parts of this plant contain immense medicinal properties thus; it is used as stimulant, restorative, male contraceptive, anti-tumor, antioxidant, antimicrobial, anti-leukemic, anti-bacterial, insecticidal and insect repellent agent respectively $[13,14]$. Besides, the roots of this plant are used in gastrointestinal disorders especially to stop dysentery and the tender shoots of this plant was used to cure mouth ulcer. Further, the paste of bark was applied with mustard oil to kill lice in the hair and the pulverized leaves are given in milk for children as a vermifuge. On the pace of routine practice, a decoction of the leafy twigs was used as a mouth-wash to relieve toothache followed by the use of ash of leaves for the healing of sores and wound gives cooling effect. In addition, the leaves were burnt and mixed with ghee to form an ointment used to heal painful ailments [6, 15]. The raw tender leaves were in the treatment of jaundice and the fruits are used in medicines as blood purifier.

\subsection{Drosophila Melanogaster: As Model Organism}

Further, in order to determine the modulatory effect of leaf extract of Maytenus emarginata (M) on paraquat induced oxidative impairments and locomotors dysfunctioning, the paraquat of required paradigm was prepared. Paraquat $(1,1-$ dimethyl-4,4-bipyridynium dichloride) is a quaternary nitrogen herbicide and highly toxic substance for humans and animals; many cases of acute poisoning and death have been reported [16]. The toxicity of paraquat is due to the generation of the superoxide anion which can lead to the synthesis of more toxic reactive oxygen species (ROS) such as hydroxyl radicals and hydrogen peroxide [17]. The use of antioxidant compounds provides an easy and convenient way of testing the validity of the free radical theory using Drosophila melanogaster as a model organism. The organisms are easier to culture and manipulate under laboratory conditions than the mammals. The administration of the test compounds can be done easily by adding the dissolved compounds to the food medium thus ensuring their uptake by the flies [18].

\subsection{Objective of Research}

In spite of published reports on plants oriented drugs and their practices in the traditional system for various serious ailments \& disorders in terms of widespread use as extracts, decoction, crude fractions, herbal tonic, the screening for neuroprotective activity remains poorly investigated. In view 
of the fact that, the multi-factorial causes have been recognized in the Central nervous system which may leads to neurodegenerative disorders. These disorders will require manifold drug therapy (Combined) to deal with the varied pathological aspects. Even though, there are many herbal drugs are functioning on multiple potentialities with respect to different ailments but, the novel pharmacological approach has been proposed explicitly to develop a multi-fangled herbal medicine formula to target and rectify the dysfunction sites in the brain.

As a result, the main rationale of the present investigation was to demonstrate if a treatment with a standardized extract of MEM can protect the human brain from neurodegenerative disorders by encumbering ROS levels and inhibiting the oxidative stress with the valid interference of potential ethnomedicine formula. Hence, the present study was designed to explore the screening for preliminary phyto-chemical, physico-chemical analysis followed by in vitro and in vivo analysis on anti-microbial, antioxidant activity linking with neuroprotective activities in methanol extract of Maytenus emarginata.

\section{Materials and Methodology}

The experimental work was carried-out in bulkiness of screening of ethno-medicinal plant drugs being practiced by Tribal healers of Biligirirangana Hills (Karnataka), India during 2011-2013 at ethno-medicinal plant research division of Hamsageetha Research Foundation, B. H. Road, Tumkur572 102, Karnataka, India. The supplementary analytical works were executed at Maharaja Institute of Technology Thandavapura-571 302, Nanjanagud taluk, Mysuru district (Karnataka), India.

\subsection{Collection of Plant Drug Material and Extract Preparation}

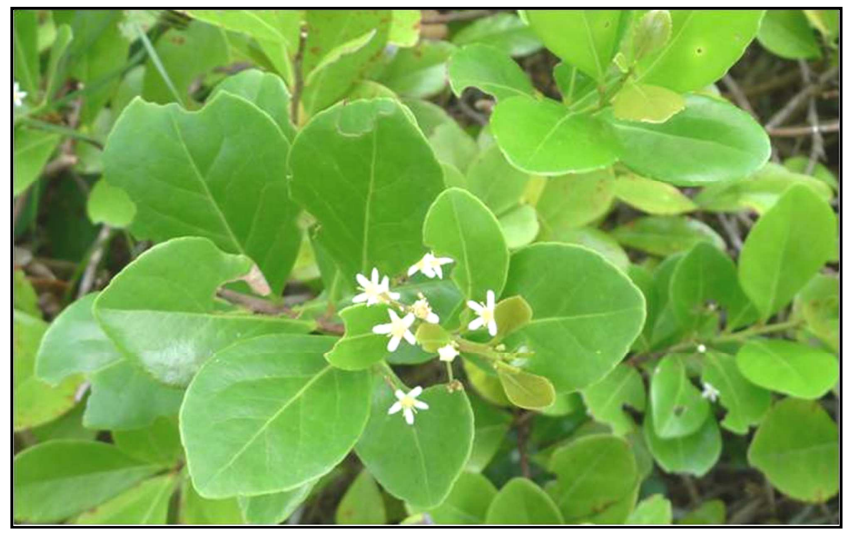

Figure 1. Maytenusemarginata (Wild.) Ding Hou- $A$ view.

The Maytenus emarginata was collected from tribal medicine men through semi-structured interactions held at Biligirirangana hills, Karnataka, India (Figure 1). Further, the leaves were collected and rinsed with distilled water and the samples were dried for 5 days in the dark at room temperature. After five days, they were oven-dried for 1 hour at $60^{\circ} \mathrm{C}$ and then subjected for grinding to obtain fine powder using mechanical blender. Later, the crude extract was prepared in the methanol and the same was filtered through a Whatman No. 1 filter paper. Finally, the extract was evaporated under reduced pressure using rotary evaporator and the powder was stored in dark glass bottles for further use.

\subsection{Physico-Chemical Characterization and Preliminary Phyto-chemical Analysis}

The Physico-chemical analysis was evaluated as per standard procedure in which total ash, acid-insoluble, ash water soluble, foreign organic matter, determination of Moisture content assays for methanol extract [19].

The Phyto-chemical screening of the Methanol extract of Maytenus emarginata was carried out to identify the major constituents in the sample. The standard procedure was used to determine phyto-chemical properties [20].

\subsection{Antimicrobial Studies}

The Methanol leaf extract of Maytenus emarginata (MEM) were screened for their antifungal activity against Aspergillus niger, Candida albicans. Subsequently, the antibacterial property was evaluated against Staphyllococcus aureus, Salmonella typhi, and Bacillus subtilis strains by disc diffusion method. The compounds were dissolved in DMSO and antimicrobial activity was determined by serial plate dilution method [21].

\subsection{In vitro Antioxidant Activity}

DPPH Radical scavenging activity

The DPPH assay was carried out according to the standard method with some modifications. The stock solution was prepared by dissolving $24 \mathrm{mg}$ DPPH with $100 \mathrm{~mL}$ methanol and then stored at $-20{ }^{\circ} \mathrm{C}$ until needed. The working solution was obtained by mixing $10 \mathrm{~mL}$ stock solution with $45 \mathrm{~mL}$ methanol to obtain an absorbance of $1.17 \pm 0.02$ units at 517 $\mathrm{nm}$ using the spectrophotometer. The solution of compounds at $5 \mu \mathrm{g} / \mathrm{ml}$ concentration was prepared in methanol. The different aliquot of stock solution was used for estimation. It was diluted to $4 \mathrm{ml}$ using distilled water. To this $1 \mathrm{ml}$ of $1,1-$ diphenyl-2-picryl-hydrazyl (DPPH) solution in methanol was added. The mixed solution was incubated at room temperature for $30 \mathrm{~min}$. The absorbance of stable DPPH • was read at $517 \mathrm{~nm}$ using UV-vis. spectrophotometer and the remaining DPPH was calculated [22].

\subsection{Preparation of Drosophila Model System for Neuroprotective Studies}

a) Wheat Cream Agar Medium and Culturing of Flies

D. melanogaster (Oregon K) adult males (8-10 days old) were obtained from Drosophila stock centre, Department of Studies in Zoology, University of Mysore, Manasagangotri, Mysore, Karnataka, India. The flies were maintained at 22 
$\pm 1^{\circ} \mathrm{C}$ and $70-80 \%$ relative humidity, fed on a standard wheat cream agar medium seeded with yeast. The medium was prepared according to standard protocol of the media (100 mL) containing $10 \mathrm{~g}$ wheat flour, $10 \mathrm{~g}$ jaggery, $1 \mathrm{~g}$ agar agar and $0.75 \mathrm{ml}$ propionic acid (antifungal agent) few granules of yeast were added. After 24 hours flies were transferred to fresh media bottles to avoid sticking of flies to media. Whenever required, the flies were exposed to the fumes of diethyl ether in a small airtight glass container for 1 min. for observation under stereo-zoom and for other studies.

b) Preparation of extract for feeding the Flies

Plant extract $(\mathrm{M})$ was dissolved in methanol. The $\mathrm{M}$ was introduced into the medium at semisolid state and mixed well then allowed to solidify. Adult males (50no.) were introduced into the vials containing media. The safety Evaluation of $\mathrm{M}$ was dissolved in $\mathrm{CH}_{3} \mathrm{OH}$. The toxicity of $\mathrm{CH}_{3} \mathrm{OH}$ was checked in the medium by rearing the flies in media with and without $100 \mu \mathrm{L}$ Et-OH. Since there was no mortality in the flies reared on medium containing $100 \mu \mathrm{L} \mathrm{CH}_{3} \mathrm{OH}$, for all experimental purposes wheat cream agar medium was used with $0.5 \%$ DMSO. Further studies were carried out to find out whether the compounds are causing mortality in the experimental batches. In this set of experiments, the males (test) were fed on a medium containing M 10 and $20 \mu \mathrm{g} / \mathrm{mL}$ concentrations. In each vial $4 \mathrm{~mL}$ of control food or food containing the compounds were added. The vials were closed with cotton stoppers. Then the lethality due to compounds was monitored by counting dead flies for every $24 \mathrm{~h}$ up to 7 days and data was expressed in terms of percentage mortality [23].

\section{c) Whole Body Homogenate Preparation}

Sodium-phosphate buffer 0.1M, ( $\mathrm{pH} 7.4)$ was used for preparing whole body homogenates. The males (30 no.) from control and tested groups were used for this purpose. After homogenizing, the samples were centrifuged at $2500 \mathrm{X} \mathrm{g}$ for $12 \mathrm{~min}$ at $4^{\circ} \mathrm{C}$. The supernatant was filtered through nylon mesh (pore size, $10 \mu \mathrm{m}$ ) and used for biochemical assays [24].

d) Paraquat Exposure and Concentrations

In a preliminary study, flies were exposed to paraquat at concentrations of $10,15,20$ and $25 \mathrm{mM}$ for $96 \mathrm{~h}$ to determine lethality. However, to assess the antioxidant effect of $\mathrm{M}$, only one concentration of Paraquat $(15 \mathrm{mM})$ was employed. For these studies, paraquat exposed flies were provided with $M$ $(20 \mu \mathrm{g} / \mathrm{mL})$ in the diet and we determined the modulatory effect of $\mathrm{M}$ on paraquat induced oxidative impairments and locomotors dysfunctioning.

\subsection{Typical Exposure Protocol and Paraquat Resistance Test}

For mortality studies, a minimum of 50 adult flies per replicate (three replicates) were exposed to $\mathrm{M}$ at $20 \mu \mathrm{g} / \mathrm{mL}$ or Paraquat $15 \mathrm{mM}$ or a combination of $\mathrm{M}+$ Paraquat. Lethality of the flies was monitored for $96 \mathrm{~h}$ and data was expressed in terms of percent mortality.

For Paraquat Resistance Test, two days after emergence from the pupae, male flies were fed with control food or food containing the compounds for a period of 7 days. Then 50 of the flies fed with the test $\mathrm{M}$ and the control were starved for 6 $\mathrm{h}$ to be sure that no food remained in the digestive tract so that none of the compounds would alter the uptake of paraquat. Afterwards, the flies were transferred to vials containing only filter paper soaked with $15 \mathrm{mM}$ paraquat in $5 \%$ sucrose solution. This concentration of paraquat was selected because it let us to study the flies for a period of $24 \mathrm{~h}$ or more after treatment. Survival was determined 24 and $48 \mathrm{~h}$ later. Survived flies were used for homogenization for biochemical assays. Each assay was repeated thrice [24].

\subsection{Biochemical Investigations}

\section{a) Assay for Lipid Peroxidation}

Lipid peroxidation was measured by employing thiobarbituric acid (TBA). Briefly, the reaction mixture containing $500 \mathrm{~mL}$ fly homogenate, $1.5 \mathrm{~mL}$ acetic acid ( $\mathrm{pH} 3.5$, $20 \%), 1.5 \mathrm{~mL}$ of TBA $(0.8 \% \mathrm{w} / \mathrm{v}), 200 \mathrm{~mL}$ sodium lauryl sulphate (SDS) $(8 \% \mathrm{w} / \mathrm{v})$. The mixture was heated in a boiling water bath for $45 \mathrm{~min}$ and adducts formed were extracted into $3 \mathrm{~mL}$ of 1-butanol. The absorbance was measured at $532 \mathrm{~nm}$ and quantified as malondialdehyde equivalents using 1,1,3,3tetra methoxypropane as the standard [25].

b) Assay for Hydroperoxide

Hydroperoxide generation was determined according to the method proposed by Wolf (1994). An aliquot of homogenate (100mg protein) was added to $1 \mathrm{~mL}$ of FOX reagent $(250 \mathrm{mM}$ Ferrous ammonium sulphate; $100 \mathrm{mM}$ sorbitol; $25 \mathrm{mM} \mathrm{H}_{2} \mathrm{SO}_{4} ; 100 \mathrm{mM}$ xylenol orange), and incubated for $30 \mathrm{~min}$ at room temperature. The absorbance was taken at $560 \mathrm{~nm}$ and expressed as $\mathrm{nm}$ $\mathrm{HP} / \mathrm{mg}$ protein.

\subsection{Estimation of Reduced Glutathione}

Reduced glutathione (GSH) content was estimated based on a fluorimetric method [26] employing o-phthalaldehyde (OPT). An aliquot of homogenate (stable reduced glutathione with $0.1 \mathrm{M}$ formic acid, $5200 \mathrm{X} \mathrm{g}$ for $10 \mathrm{~min}$ ) was allowed to react with OPT $(1 \mathrm{mg} / \mathrm{mL}$ in methanol) at room temperature for $30 \mathrm{~min}$ and fluorescence measured at excitation of 345 $\mathrm{nm}$ and emission at $425 \mathrm{~nm}$.

\subsection{Activities of Antioxidant Enzymes}

Catalase activity was determined according to a standard protocol [27]. Accordingly, to $1 \mathrm{~mL}$ reaction mixture containing $8.8 \mathrm{mM} \mathrm{H} \mathrm{H}_{2} \mathrm{O}_{2}(3 \%), 0.1 \mathrm{mM}$ sodium-phosphate buffer, $\mathrm{pH} 7.0$ the reaction was initiated by adding an aliquot (equivalent to $10 \mathrm{mg}$ protein). The decrease in $\mathrm{H}_{2} \mathrm{O}_{2}$ was monitored for $3 \mathrm{~min}$ at $240 \mathrm{~nm}$.

SOD activity was determined by monitoring the inhibition of quercetin auto oxidation. Total volume of $1 \mathrm{~mL}$ reaction mixture containing 3-5 mg protein; $0.016 \mathrm{M}$ sodium phosphate buffer, $\mathrm{pH} 7.8 ; \mathrm{N}, \mathrm{N}, \mathrm{N}, \mathrm{N}$ Tetramethy 1 ethylene diamine (TEMED), $8 \mathrm{mM}$ and ethylene diamine tetra acetic acid (EDTA), $0.08 \mathrm{mM}$ and reaction was started by adding $0.15 \%$ quercetin dissolved in dimethyl formamide. Reaction was 
monitored for $3 \mathrm{~min}$ at $406 \mathrm{~nm}$, expressed as amount of protein required to inhibit $50 \%$ of quercetin auto oxidation [28]. The protein estimation was done using flies' homogenate as per the standard procedure [29].

\section{Results and Discussion}

The methanol extract $M$ was characterized initially for both physical and phyto-chemical potentials. The phytochemical studies reveal that, the extract $\mathrm{M}$ was associated with flavonoids, phenols and other components. The data is illustrated in the Tables 1 and 2 respectively.

Table 1. Physico chemical Characterizations of methanol extract of Maytenus emarginata.

\begin{tabular}{lll}
\hline Sl. No & Parameters & Average value(\% w/w) \\
\hline 1 & Total ash & $58.6 \%$ \\
2 & Acid insoluble ash & $71.33 \%$ \\
3 & Water soluble & $77.25 \%$ \\
4 & Foreign organic matter & $6.5 \%$ \\
\hline
\end{tabular}

Table 2. Phyto-chemical analysis of methanol extract of Maytenusemarginata.

\begin{tabular}{lll}
\hline Sl. No & Class of constituents & Remarks \\
\hline 1 & Carbohydrates & + \\
2 & Proteins & + \\
3. & Alkaloids & - \\
4. & Phenolic compounds & + \\
5. & Saponins & - \\
6. & Flavonoids & + \\
\hline
\end{tabular}

The in vitro antioxidant assays like DPPH and TBARS assay were carried out. The extract $M$ exhibited better antioxidant activity in concentration dependent manner. The data is depicted in the Figures 2 and 3 respectively.

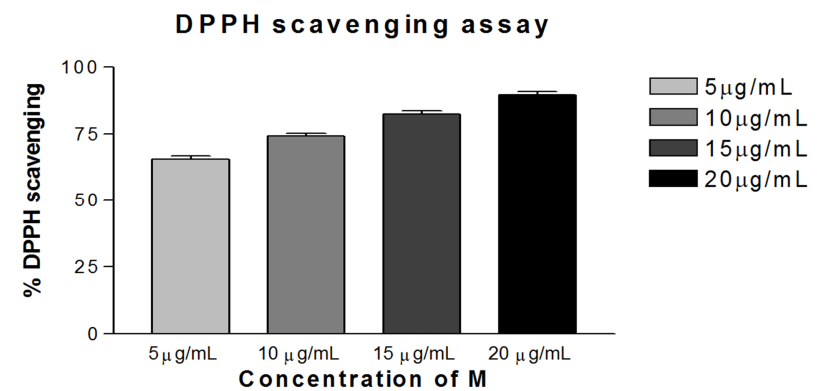

Figure 2. In vitro DPPH scavenging activity M. Result is expressed as \% $D P P H \cdot$ scavenged.

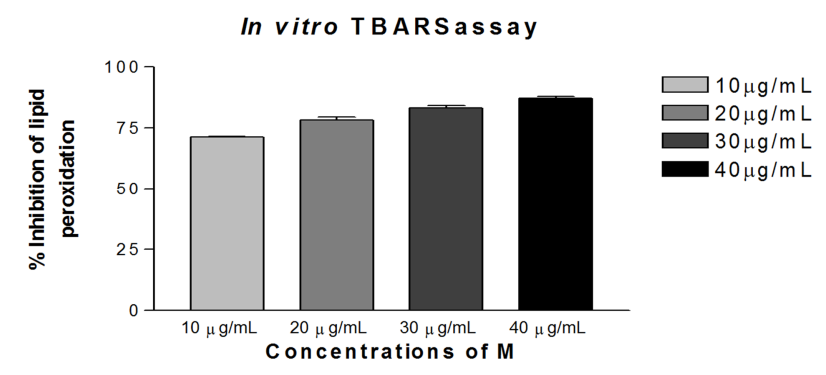

Figure 3. In vitro lipid peroxidation assay of $M$.

The main aim was to elucidate antioxidant and preliminary neuroprotective efficacy of the $\mathrm{M}$ in vivo using the organism; Drosophila melanogaster in this study because it is an excellent model for gerontological research due to its relatively short life span; the adult flies appear to show many of the manifestations of cellular senescence observed in mammals and oxidative stress plays an important role in governing the life span of the fly. Toxicity study was done to determine the lethal concentration of M. Safety evaluation of $M$ revealed that the $M$ was non toxic at experimental concentration which is given in Table 3.

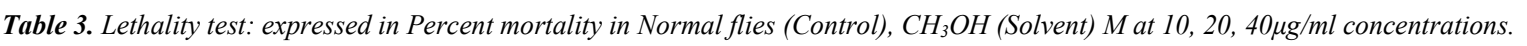

\begin{tabular}{|c|c|c|c|c|c|c|c|c|}
\hline \multirow{3}{*}{$\begin{array}{l}\text { Standard } \\
\text { media } \\
\text { Control } \\
\end{array}$} & \multirow{2}{*}{ Conc. } & \multicolumn{7}{|l|}{ Days } \\
\hline & & 1 & 2 & 3 & 4 & 5 & 6 & 7 \\
\hline & ---- & 0.0 & 0.0 & $0.26 \pm 0.05$ & $0.26 \pm 0.05$ & $0.16 \pm 0.05$ & $0.23 \pm 0.05$ & $0.33 \pm 0.05$ \\
\hline \multirow[t]{2}{*}{$\mathrm{CH}_{3} \mathrm{OH}(\mu \mathrm{l})$} & 100 & $0.466 \pm 0.32$ & $0.61 \pm 0.25$ & $0.64 \pm 0.31$ & $0.83 \pm 0.20$ & $0.96 \pm 0.20$ & $1.04 \pm 0.20$ & $1.43 \pm 0.26$ \\
\hline & 10 & $0.36 \pm 0.25$ & $0.56 \pm 0.40$ & $0.63 \pm 0.36$ & $0.83 \pm 0.36$ & $1.38 \pm 0.21$ & $1.12 \pm 0.19$ & $1.73 \pm 0.37$ \\
\hline \multirow[t]{2}{*}{$\mathrm{M}(\mu \mathrm{g} / \mathrm{ml})$} & 20 & $0.46 \pm 0.15$ & $0.56 \pm 0.25$ & $0.76 \pm 0.15$ & $0.93 \pm 0.25$ & $1.50 \pm 0.26$ & $1.30 \pm 0.17$ & $1.80 \pm 0.10$ \\
\hline & 40 & $0.58 \pm 0.20$ & $0.76 \pm 0.30$ & $0.96 \pm 0.30$ & $1.41 \pm 0.20$ & $1.59 \pm 0.17$ & $1.52 \pm 0.17$ & $1.83 \pm 0.15$ \\
\hline
\end{tabular}

The extract $\mathrm{M}$ was also screened for their antibacterial activity against Escherichia coli K-12, Staphyllococcus aureus, Salmonella typhi, and Bacillus subtilis. It was also screened for their antifungal activity against Aspergillus niger and Candida albicans. The extract $\mathrm{M}$ exhibited significant activity against tested organisms However, based on this promising observation; it is immature to arrive at the conclusion. The zone of inhibition and the minimum inhibitory concentration (MIC) values of the compounds tested against bacteria and fungi are given in the Table 4.

Table 4. Antimicrobial activity of $M$.

\begin{tabular}{|c|c|c|c|c|c|c|}
\hline Extract & Staphylococcus aureus & $\begin{array}{l}\text { Bacillus } \\
\text { subtilis }\end{array}$ & Salmonella typhi & Escherichia coli & Aspergillus niger & $\begin{array}{l}\text { Candida } \\
\text { albicans }\end{array}$ \\
\hline $\mathrm{M}$ & $26(10)$ & $24(10)$ & $20(20)$ & $28(10)$ & $24(30)$ & $12(40)$ \\
\hline Standard & 24 & 23 & 23 & 25 & 25 & 24 \\
\hline DMSO(Control) & 0 & 0 & 0 & 0 & 0 & 0 \\
\hline
\end{tabular}

Note: - Standard drug used: Bacteria (Ciprofloxcin), Fungal (Fluconazole) (40 $\mu \mathrm{g}$ in $100 \mu \mathrm{L})$.

M used: (40 $\mu \mathrm{g}$ in $10 \mathrm{~mL}$ - based on MIC concentration). Control: DMSO (dimethyl sulphoxide)

Zone of Inhibition in $\mathrm{mm}$ : MIC in $\mu \mathrm{g} / \mathrm{mL}$ (data given in the parenthesis is for MIC). 
The different concentrations of Paraquat were used to determine concentration and time dependent mortality of flies. Concentration and time dependent mortality response of paraquat on adult male Drosophila melanogaster was given in the Figure 4. Result reveals that, there was high mortality (50-60\%) among flies during a 7-day exposure period with Par. Where as in case of co exposure of M and Par showed significant reduction in mortality rate. The solvent methanol $(100 \mu \mathrm{l})$ was kept as solvent control. In this case also mortality rate is insignificant compared to Par treated flies.

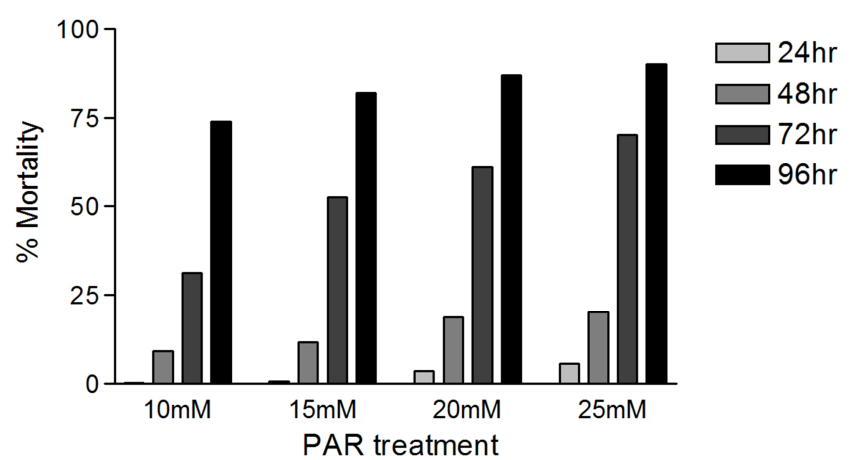

Figure 4. Concentration and time dependent mortality response among adult male Drosophila melanogaster exposed to PAR in the feed. $(n=50$ flies per replicate, three such replication used for assay).

The incidence of mortality among adult male Drosophila melanogaster exposed to paraquat, is considerably high where as there was a decrease incidence of mortality among flies exposed to $M+$ PAR, This results support that $M$ showed protective action. The data is given in the Figure 5. The above results instigated us to determine antioxidant effect of $\mathrm{M}$ against paraquat induced oxidative stress.

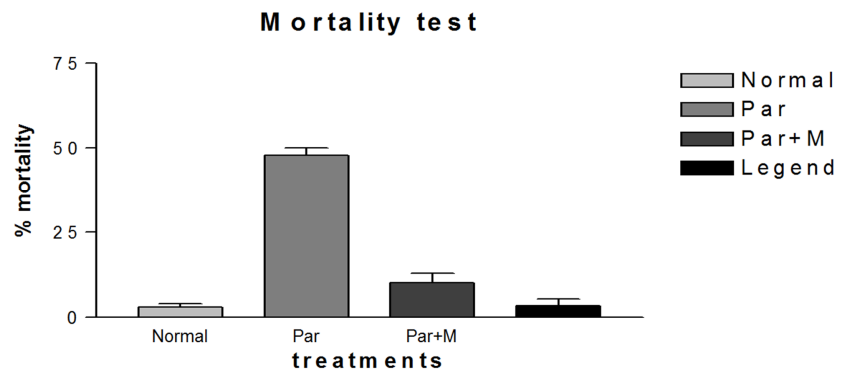

Figure 5. Incidence of mortality among adult male Drosophila melanogaster exposed to Paraquat, co-exposure asM+PAR; note: decrease incidence of mortality among flies co-exposed with $M+P A R$.

It was reported that, the significant induction of oxidative stress among flies exposed to paraquat $15 \mathrm{mM}$ evidenced by the marked elevation in MDA and hydroperoxide level coupled with significant change in the activities of antioxidant enzymes such as CAT, SOD which suggested an increased generation of ROS. Ameliorative effects of $M$ on paraquat induced oxidative stress markers in whole body homogenates of adult flies fed with M supplemented diet for 7 days showed significant diminution in MDA and HP levels. The data is given in Figure 6 and 7 respectively.

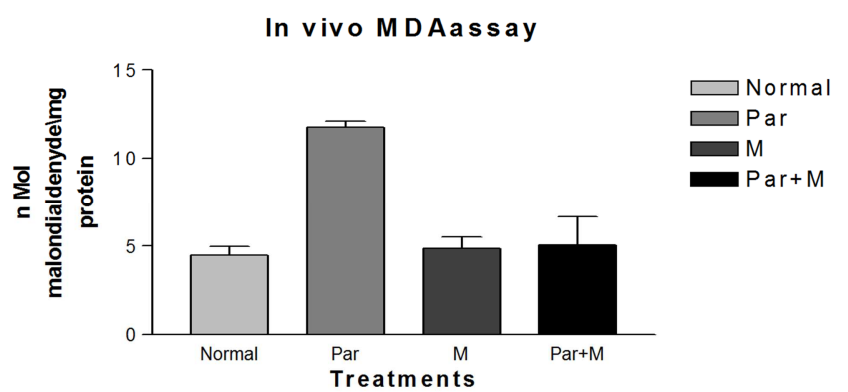

Figure 6. Ameliorative effect of $M$ treatment on paraquat (PAR) induced oxidative stress measured asmalondialdehyde levels in whole body homogenates of adult Drosophila melanogaster.

\section{In vivo HP studies}

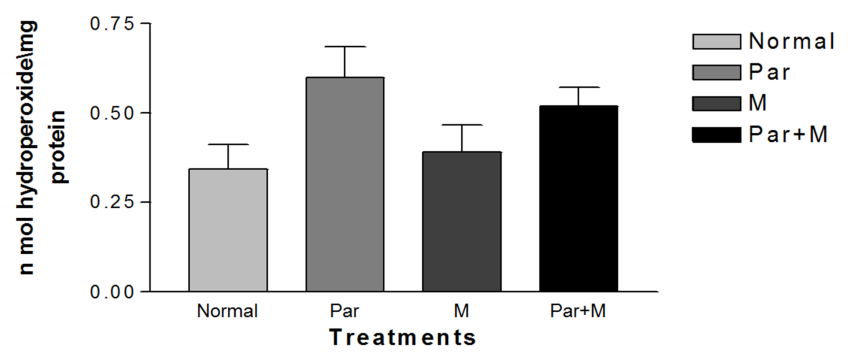

Figure 7. Ameliorative effect of $M$ treatment on paraquat (PAR) induced oxidative stress measured as hydroperoxide levels in whole body homogenates of adult Drosophila melanogaster.

The reduced GSH is a tripeptide and most abundant soluble antioxidant molecule which plays a crucial role in detoxifying ROS either by reacting directly with radicals non-enzymatically or acting as a substrate in GST catalyzed reactions. Severe depletion in cellular GSH levels upon paraquat exposure in Drosophila adds further evidence that a state of oxidative stress exists in vivo which may lead to mitochondrial damage, increase in free radical generation and peroxidation of membrane lipids. Although paraquat exposure for 7 days caused significant decrease in GSH level, on co exposure with $\mathrm{M}$, flies were able to restore the depleted GSH levels significantly Figure 8 Clearly suggesting the ability of M to up regulate levels of GSH.

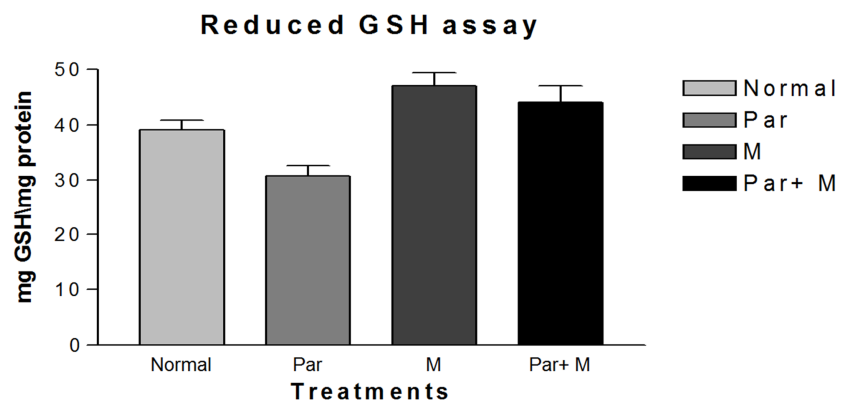

Figure 8. Ameliorative effect of $M$ treatment on paraquat (PAR) induced oxidative stress measured asreduced glutathionelevels in whole body homogenates of adult Drosophila melanogaster.

The Parquat $(15 \mathrm{mM})$ exposure induced a significant elevation in the activity of all antioxidant enzymes measured. 
However, among $\mathrm{M}+$ paraquat exposed flies, the activity levels of CAT and SOD, were restored to near basal levels, Figures $9 \& 10$.

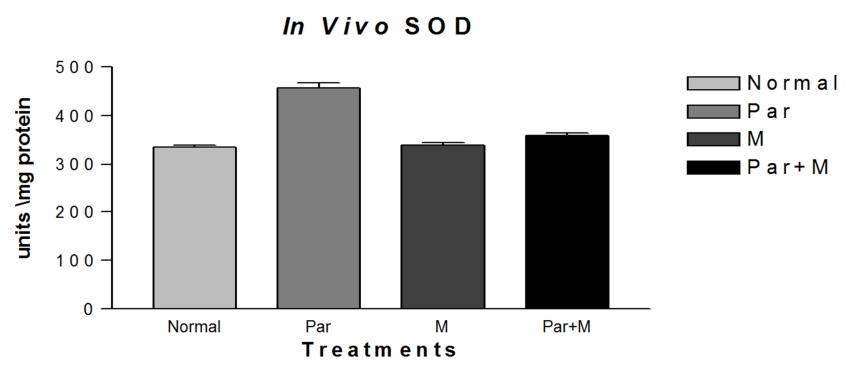

Figure 9. Modulatory effect of $M$ on the endogenous markers of oxidative stress SOD in adult Drosophila melanogaster.

In Vivo CAT assay

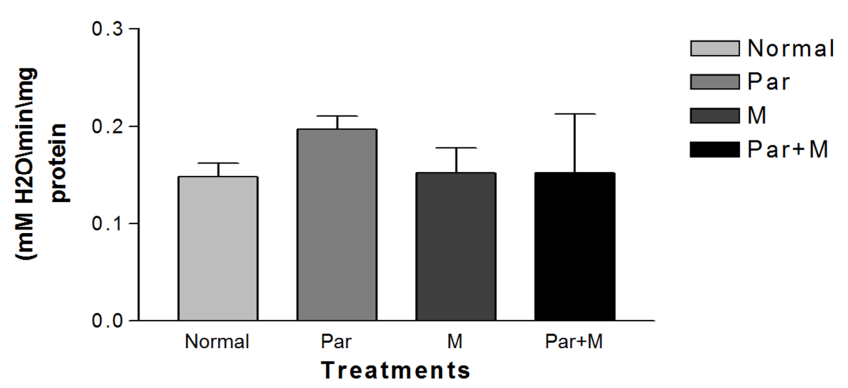

Figure 10. Modulatory effect of $M$ on the endogenous markers of oxidative stress HP in adult Drosophila melanogaster.

Based on biochemical evidences, we propose that dietary feeding of leaf extract of Maytenus emarginata (M) to Drosophila for a short duration has the propensity to attenuate paraquat induced oxidative stress owing to its antioxidative nature and its ability to modulate the activities of antioxidant defenses such as reduced GSH and antioxidant defenses. Additional evidences viz., lower incidence of paraquat induced mortality and higher resistance to paraquat among flies pretreated with M. Further, its antioxidant property was clearly evident by its ability to significantly abrogate paraquat induced oxidative stress, by depleting the lipid peroxidation product malanoldialdehyde.

In the system, the proteins and lipid are measured as most vital macromolecules when these are damaged by paraquat induction which results in the meddling with regular functioning of cell organelles like mitochondria and lysosomes making them less competent with respect to their normal function. The extract of $\mathrm{M}$ may have ability to protect these macromolecules and thereby showed the evidence of neuroprotection. Therefore, the conservation of endogenous antioxidant with the treatment of defensive enzymes of MEM extract further indicated the neuroprotection from the free radical induced toxicity. The similar findings are also reported [30].

Subsequently, the biochemical investigation revealed the significant increase in the lipid peroxidation, superoxide dismutase activity and fall in total suphahydral (T-SH) levels which is an indicative of antioxidant properties of MEM extract in neuroprotection [31]. The subsequent study with methanolic extract of WS also reported significant inhibition of AchE activity and thereby suggested its role in AD prominently associated with cholinergic depletion. The collective data supports the use of WS as a neuroprotective with putative mechanism of action [32].

Further, the amelioration indicated that the possible cholinergic modulation as mechanism of its action and thus designated possible utility in the management of AD and age associated dementias [33, 34]. Hence, in the present investigation, momentous approach was observed in the MEM plant drug and was substantiated by the earlier findings with respect to progression in the neurobehavioral performance associated with significant reduction in malonaldehyde levels in cortex and increased in glutathione as well as superoxide dismutase activity in cortex and corpus striatum in rats $[4,35,36,37]$.

The results obtained in 'paraquat resistance assay' suggest that $\mathrm{M}$ prophylaxis has the propensity to protect against neurotoxicant exposure largely due to its antioxidative potential. In this model, the protective activity of $M$ was highly comparable to paraquat alone treated [38]. More importantly this data further confirm the utility value of Drosophila system as a primary model to rapidly screen potential compounds for their antioxidant properties prior to their testing in mammalian models and final therapeutic use in humans [39, 40].

In all, the MEM plant drug has shown beneficial effects on cognitive function. However further studies on accomplishing active lead molecules responsible for the proper mechanism involved is found to be very much necessary. In addition, the more distinctive ethno-medicinal plant drugs with multi-fangled properties via scientific validation for the treatment of a neurodegenerative disease are most required. The coherent protocol has to be standardized to develop promising drug of more acceptable and better substitute to the present pharmacotherapy.

Future scope of the Research

At the outset, the result generated from the present research revealed that, the antioxidant property of MEM plant drug may provide most prospective therapeutic intercession against oxidative menace associated with and neurodegenerative disorders and can be considered as most potent nutraceutical candidate plant drug. Therefore, the outcome of the study can also justify the practices of MEM plant drug by 'tribal medicine men' in their traditional system for early aging and neurodegenerative related disorders. However, by taking into consideration of the above information's correlated with present findings, the subsequent evaluation is also required to explore the actual lead molecules involved in the therapeutic system with probable mechanism.

\section{Conclusion}

It is evident from the experimental results that, the active 
principle of Maytenus emarginata M is good antioxidant, antimicrobial against tested organisms and neuroprotective agent via Drosophila model system. This work specifically opens up an avenue for further investigation towards the characterization of lead constituents and evaluation of biological properties with molecular mechanism.

In addition, there are still petite upshots on breakdown for explicit assays are needed to examine their potential neuroprotective properties. The assessment based on genomics and proteomics are expected to offer cumulative information's about molecular mechanism of neurological ailments and their protection by extracts of ethno-medicinal plant with wide ranging point of views. This will facilitate the potential applications of active principle to the highest degree by emerging as a newfangled drug for the prevention of neurodegenerative disorders.

\section{Conflict of Interest}

Authors declare that there are no conflicts of interest.

\section{References}

[1] Kumar, G. P. and Khanum, F. Neuroprotective potential of phytochemicals, Pharmacognosy Reviews, vol. 6, no. 12, pp. 81- 90, 2012

[2] Ekor, M. The growing use of herbal medicines: Issues relating to adverse reactions and challenges in monitoring safety," Frontiers in Neurology, vol. 4, p. 177, 2014.

[3] Harvey, A. L. Natural products in drug discovery," Drug Discovery Today, vol. 13, no. 19-20, pp. 894-901, 2008.

[4] Limpeanchob N, Jaipan S, Rattanakaruna S, Phrompittayarat W, Ingkaninan K. Neuroprotective effect of Bacopamonnieri on beta-amyloid-induced cell death in primary cortical culture, Naresuan University, Phitsanulok 65000, Thailand Journal of Ethnopharmacology 2008; 120: 112-117.

[5] Finkel, T, Holbrook, NJ. 2000. Oxidants: oxidative stress and the biology of ageing. Nature, 408: 239-247.

[6] Kothari, M. J and A. N Londhe. 2000. Ethno-botany in Human health care of Chikkhaldara, Amravati district in maharashtra state, India. Ethnobotany and medicinal plants of Indian subcontinent, Scientific publishers (India) Jodhpur, 273-281.

[7] Panduranga Murthy, G. Chandrasekhar, K. B. Ravishankar, D. K. Leelaja, B. C. Lokesh, S. Manju. B. 2017. Evaluation of Validated Herbal Medicine Formulation for Anti-Proliferative and Apoptotic Activities Used In the Tribal Medicine System, IOSR Journal of Applied Chemistry (IOSR-JAC); V-10(1) Pp: 05-24.

[8] WHO. WHO Guidelines on Safety Monitoring of Herbal Medicines in Pharmacovigilance Systems, World Health Organization, Geneva, Switzerland, 2004.

[9] Panduranga Murthy G., Harsha, R., Leelaja, B. C., Chandrasekhar, K. B and Lokesh. 2016. Snake venom neutralizing effect of validated Herbal Medicine Formula Practiced in Tribal Medicine System (TMS) at B. R. Hills region of Karnataka, India. Int. J. Res. Pharm. Sci.7(3)2; 225244.

[10] Panduranga Murthy G., Chandrasekhar K. B and Lokesh S. 2015. Evaluation of Ethno-medicinal plant drugs for Wound healing practiced by tribal healers of Biligirirangana Hills (Karnataka), India. World Journal of Pharmacy and Pharmaceutical Sciences; Vol-4(05): 2035-2058.

[11] Nadkarni, K. M.: Indian MateriaMedicaVol 1\&II., Popular Prakashan Pvt. Ltd, Mumbai (1976).

[12] Takimoto, C. H., E. Calvo. 2008. Principles of Oncologic pharmacotherapy inpazdur R, Wagman LD, Camphausen KA, Hoskins WJ [EDS] Cancer management: A multidisciplinary Approach. 11ed.

[13] Gonzalez, J. G., G. D. Monache, F. D. Monache, and G. B. Marini Bettolo. 1982. J. Ethnopharmacol., 5, 73.

[14] Itokawa, H., O. Shirota, H. Morita, K. Takeya, and Y. Iitaka. 1991. Phytochemistry, 30, 3713.

[15] Pullaiah T. 2006. Encyclopedia of world medicinal plants, Sal. Paratyphi Regency publication, $1^{\text {st }}$ Edn. New delhi, PP:13161317.

[16] Sittipunt, C. 2005. Paraquat poisoning. Respir Care., 50: 383385.

[17] Suntres, ZE. 2002. Role of antioxidants in paraquat toxicity. Toxicol., 180:65-77.

[18] Tapiwanashe, M, Melanie, W, Kumars, R, Michael, PM, Robin, AJ, Smith, LP. 2006. The effects of exogenous antioxidants on lifespan and oxidative stress resistance in Drosophila melanogaster. Mech. Age. Devpt., 127: 356-370.

[19] Khandelwal K. R., Practical pharmacognosy Techniques and Experiments, NiraliPrakashan, 1999; (146-148).

[20] Sazadasiddiqui, Artiverma, Ayaz Ahmad Rather, FarahaJabeen and Mukesh K. Meghvansi. 2009. Preliminary Phyto-chemical Analysis of some Important Medicinal and Aromatic plants. Advances in Biology Research. 3 (5-6):188195.

[21] Nada MA, Hamdi MH, Nadia GK, Omar AM. 2008. Synthesis and Antimicrobial Activity of Some New Pyrazole, Fused Pyrazolo[3,4-d]-pyrimidine and Pyrazolo[4,3-e][1,2,4]Triazolo [1,5-c] pyrimidine Derivatives. Molecules 13: 15011517.

[22] Sarojini, BK, Darshan Raj CG., Ramakrishna, MK, Ramesh SR, Bharath, BR, Manjunatha, H. 2011. In Silico Studies of (2E,5E)-2,5-bis(3-methoxy-4-hydroxy-benzylidene) Cyclopentanone on Proteins $\mathrm{AChE}$ and $\mathrm{BChE}$ Involved in Alzheimer's disease and Ameliorative Effects on Paraquat Induced Oxidative Stress Markers in Drosophila melanogaster. Lett. in DrugDes. \& Disc., 8: 260-267.

[23] Ravikumar, H., Muralidhara. 2009. Neuroprotective efficacy of Bacopamonnieri against rotenone induced oxidative stress and neurotoxicity in Drosophila melanogaster. NeuroToxicol., 30: 977-985.

[24] Lin, A. 1998. Extended life span and stress resistance in the Drosophila mutant Methuselah. Science, 282: 943-946.

[25] Ohakawa, H, Ohishi, U, Yakgi, K. 1979. Aassay of lipidperoxidation in rat tissues by thiobarbituric reaction. Anal. Biochem., 95: 145-149. 
[26] Hissin, PJ, Hilf, R. 1976. A flourimetric method for determination of oxidized and reduced glutathione in tissues. Anal Biochem., 74: 214-216.

[27] Aebi H, (1984), Catalase in vitro. Meth. Enzymol., 105: 121125.

[28] Kakkar, BD, Viswanathan, PN. 1984. A modified spectrophotometric assay of superoxide dismutase (SOD). Ind. J. Biochem. Biophys., 21: 130-132.

[29] Lowry, OH, Rosebrough, NJ, Farr, AL, Randall, RJ. 1951. Protein measurement using Folin phenol reagent. J. Biol. Chem., 282: 943-946.

[30] Stahl, S. M.: Essential Psychopharmacology, CambridgeUniversity Press, (1998).

[31] Veerendra Kumar, M. H., Gupta, Y. K.: J. Ethnopharmacol., 79: 253-260 (2002).

[32] Vinutha, B., Prashanth, D., Salma, K., Sreeja, S. L., Pratiti, D., Padmaja, R., Radhika, S., Amita, A., Venkateshwarlu, K., Deepak, M.: J. Ethnopharmacol., 109: 359-363 (2007).

[33] Shukla, S. D., Jain, S., Sharma, K., Bhatnagar, M.: Ind J. Expt. Bio., 38: 1007-1013(2000).

[34] Kennedy, D. O., Scholey, A. B.: Pharmacol. Biochem. Behav., 75:687-700 (2003).
[35] Chaudhuri A, Bowling K, Funderburk C, Lawal H, Inamdar A, Wang Z, O'Donnell JM. Interactions of genetic and environmental factors in Drosophila Parkinsonism model. J. Neurosci., 27:2457-67.

[36] Feany, MB, Bender, WW. 2000. A Drosophila model of parkinson's disease. Natr., 404: 394-398.

[37] McCormack, AL, Atienza, JG., Johnston, LC, Andersen, JK, $\mathrm{Vu}, \mathrm{S}$, Di Monte, DA. 2005. Role of oxidative stress in paraquat-induced dopaminergic cell degeneration. J Neurochem., 93: 1030-1037.

[38] Orr, WC, Radyuk, SN, Prabhudesai, L, Toroser, D, Benes, JJ, Luchak, JM., Mocket, RJ, Rebrin, I, Hubbard, JG, Sohal, RS. 2005. Overexpression of glutamate-cysteine ligase extends life span in Drosophila melanogaster. J. Biol. Chem., 280: 37331-37338.

[39] Ravindra, PS, Shashwat, S, Suman, K. 2004. Free radicals and oxidative stress in neurodegenerative diseases: relevance of dietary antioxidants. JIACM, 5:218-225.

[40] Thiruchelvam, M, McCormack, A, Richfield, EK. Baggs, RB, Tank, AW, DiMonte, DA, Cory-Slechta, DA. 2003. Age related irreversible progressive nigrostriatal dopaminergic neurotoxicity in the paraquat and maneb model of the Parkinson's disease phenotype. Eur J Neurosci., 18: 589-600. 